\title{
AFM Force Spectroscopy and Steered Molecular Dynamics Simulation of Protein Contactin 4
}

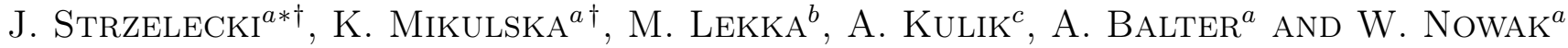 \\ ${ }^{a}$ Institute of Physics, Nicolaus Copernicus University, Grudziądzka 5, 87-100 Toruń, Poland \\ ${ }^{b}$ The Henryk Niewodniczanski Institute of Nuclear Physics, Polish Academy of Sciences \\ Radzikowskiego 152, 31-342 Kraków, Poland
}

${ }^{c}$ Institute of Physics of Complex Matter, Ecole Polytechnique Federale de Lausanne, Ch-1015 Lausanne, Switzerland

We use a single molecule atomic force spectroscopy combined with the steered molecular dynamics simulation to determine a mechanical behavior of neural cell adhesion protein contactin during its unfolding. Force curves typical for modular proteins were observed, showing at most four unfolding peaks. The analysis of force spectra performed within worm-like chain model of polymer elasticity showed the presence of three unfolding lengths. Small plateaus, most likely resulting from forced transitions within domains were observed for the first time. Steered molecular dynamics simulations help to determine atomistic picture of domain unfolding.

PACS numbers: 82.37.Rs, 87.10.Tf

\section{Introduction}

Contactins belong to the immunoglobulin super family of neuronal cell adhesion proteins. They consist of four so called FnIII and six $\operatorname{IgC} 2$ domains. The latter are augmented by disulfide bonds (Fig. 1A). Contactin4 (CNTN4, $113.454 \mathrm{kDa})$ is one of the axon guidance molecules crucial for the formation of odor map in the olfactory bulb [1]. It plays also functional roles in the formation and maintenance of neural circuits in regions like cerebellum and hippocampus. The human CNTN4 (locus $3 \mathrm{p} 26.2-3 \mathrm{p} 26.3$ ) was identified as a candidate gene responsible for $3 \mathrm{p}$ deletion syndrome, rare contiguous-gene disorder [2]. Mutations affecting CNTN4 function may be also relevant to autism spectrum disorder (ASD) pathogenesis [3]. Thus understanding nanoscale mechanical properties of contactins has a great medical significance.

Even though the atomic force microscope (AFM) was originally developed for surface imaging [4], its ability to precisely measure piconewton forces finds new scientific applications every year. This remarkable technique has already allowed to measure mechanical forces of ligand-receptor unbinding [5], intermolecular transitions in DNA [6] and polysaccharides $[7,8]$ and unfolding of proteins $[9,10]$.

Until now, only one study employing force spectroscopy to characterize human CNTN4 has been recently reported [11]. The distribution of unfolding

\footnotetext{
* corresponding author; e-mail: jast@fizyka.umk.pl

$\dagger$ These authors contributed equally to this work
}

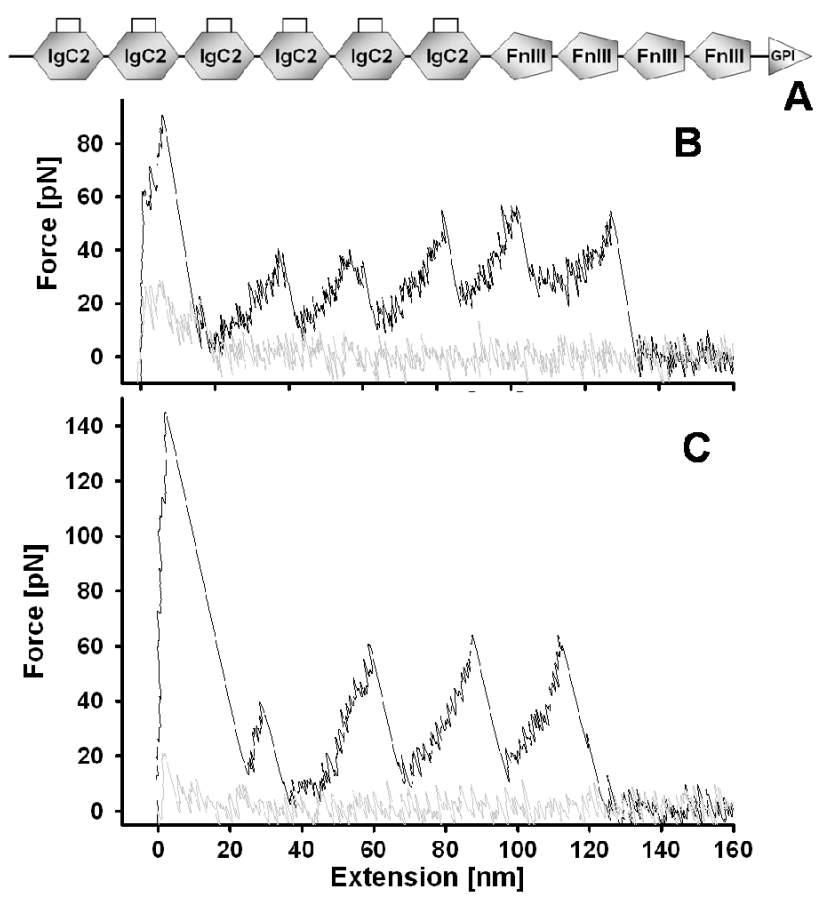

Fig. 1. Contactin 4 protein structure (A) and force spectroscopy curves $(\mathrm{B}, \mathrm{C})$.

lengths showed peaks at $18.6 \pm 5.1 \mathrm{~nm}$ and $29.2 \pm 6.7 \mathrm{~nm}$. The data suggested the existence of at least two possible unfolding events attributed to FnIII domains. The larger value corresponds to the unfolding of a whole domain, while the smaller was assigned to intermediate state unfolding. An addition of tris(2-carboxyethyl)phosphine 
(TCEP) to solution aimed at breaking of disulfide bonds resulted in an increase of number of unfolding peaks. The detailed interpretation of force spectra of contactin was difficult, due to a rather high level of noise of AFM used in the first study [11].

In our study, we apply an instrument specifically suited for a single molecule AFM force spectroscopy and with a reduced thermal noise to revisit mechanical properties of contactin. New experimental results were compared with steered molecular dynamics (SMD) simulations, reported for the first time here. As a recent study suggests a connection between contactin 4 and autism [12], we hope that better understanding of its mechanical fingerprint may open possibility of the diagnosis in a truly nanomedical fashion - one molecule at a time.

\section{Materials and methods 2.1.Sample preparations}

Human recombinant contactin CNTN4, purchased from R\&D Systems, Biokom was used. The protein was suspended in phosphate buffered saline (PBS) $(137 \mathrm{mM}$ $\mathrm{NaCl}, 2.7 \mathrm{mM} \mathrm{KCl}, 10 \mathrm{mM}$ sodium phosphate dibasic, $2 \mathrm{mM}$ potassium phosphate monobasic and a $\mathrm{pH}$ of 7.4) at a concentration of $0.025 \mathrm{mg} / \mathrm{ml}$. A drop of solution with suspended protein was placed on muscovite V1 mica freshly stripped with adhesive tape and incubated overnight in a humid atmosphere. Afterwards, the sample was placed in a liquid cell and left for $15 \mathrm{~min}$ to reach equilibrium conditions before pulling.

\subsection{The AFM force spectroscopy}

Single molecule stretching was accomplished with a custom made AFM one-dimensional puller, with a design simiar to [13]. The sample was moved vertically with a closed loop piezo actuator (Physik Instrumente, P-840.10, strain gauge sensor). Laser used for cantilever bending detection was Schäfter + Kirchhoff $\mathrm{GmbH}$ 51nanoFCM. The signal from position sensitive detector was filtered with a Bessel low pass active filter (900CT/9L8L, Frequency Devices) at $500 \mathrm{~Hz}$ during measurement and $50 \mathrm{kHz}$ during calibration.

Cantilevers used were Biolevers from Olympus Probe, with a nominal spring constant $6 \mathrm{mN} / \mathrm{m}$. Each cantilever was calibrated prior to each experiment using the thermal tune method [14]. A typical value of a cantilever force constant was within the range of $5-8 \mathrm{mN} / \mathrm{m}$.

A standard experiment involved leaving a tip pressing the sample with a force of approximately $1 \mathrm{nN}$ for several minutes to allow nonspecific tip-protein bond formation. A ramp, generated in a computer interface (National Instruments, PCI-6251) was fed to a piezo controller, causing withdrawal and approach of sample relative to a cantilever tip at $100-300 \mathrm{~nm} / \mathrm{s}$ speed. Only force curves showing at least two regular unfolding peaks were selected for further analysis. A worm-like chain model (WLC) [15] fitting was done with a PUNIAS analysis program (version 1.0 Beta 23, http://site.voila.fr/punias ).

\subsection{Steered molecular dynamics simulations}

Simulations were done with the molecular dynamics code NAMD 2.6 [16]. The model structure of human CNTN4 was solvated using $0.5 \mathrm{~nm}$ layer of TIP3P water model (7000 atoms) and equilibrated for 0.12 ns with the CHARMM22 force fields. We have run six simulations of human $\mathrm{CNTN}_{4} \mathrm{FnIII}_{3}$ domain, five for $\mathrm{CNTN} \mathrm{FnIII}_{3}$ domain (PDB code 2ee2) and four simulations of $\mathrm{IgC} 2{ }_{6}$ domain (total length $200 \mathrm{~ns}$ ). The model structures of $\mathrm{FNIII}_{3}$ (residues GLU19 to PRO119) and $\mathrm{IgC}_{6}$ (residues LYS2 to PRO103) were created by homology modeling in Swiss-Model [17] (Expasy Server http://www.expasy.ch/) based on homologous contactin 1 (2ee2 and 2edn, respectively). Constant velocity SMD method was used to stretch domains along their long axes. In this technique virtual harmonic force is applied to one end of the protein which is simultaneously fixed at the other end. The SMD method closely mimics the AFM stretching experiment [18], but the timescale of simulations, due to common hardware limitations, is $10^{4}$ time faster than the AFM experiment. Structures were stretched at a constant speed of $0.025 \AA / \mathrm{ps}$ with spring constant of $7 \mathrm{kcal} \mathrm{mol}^{-1} \AA^{-2}$. The thirteen trajectories were recorded for $15 \mathrm{~ns}$, two for $5 \mathrm{~ns}$ and analyzed with VMD 1.8.6 [19].

\section{Results and discussion}

Similarly to the previous study [11], our recorded force curves showed at most four unfolding peaks (Fig. 1B and $\mathrm{C})$.

However, an increased accuracy allowed us to use WLC polymer model fitting in order to determine unfolding lengths corresponding to consecutive peaks (Fig. 2A). A distribution of 158 unfolding lengths and 290 unfolding forces from 124 curves was plotted in histograms (Fig. 2B and $\mathrm{C}$ ). It was possible to fit 3 normal distributions, with values centered at $19.41 \pm 1.63 \mathrm{~nm}, 24.52 \pm 0.24 \mathrm{~nm}$ and $36.79 \pm 1.52 \mathrm{~nm}$.

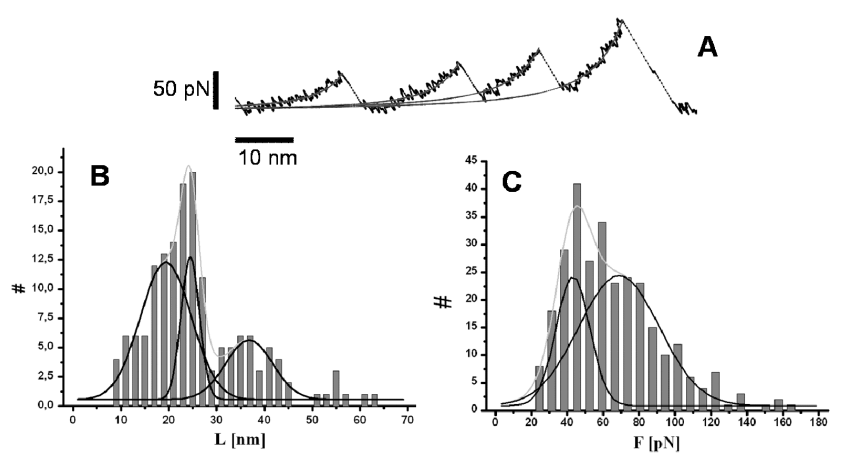

Fig. 2. An example of WLC fit (A) and distributions of unfolding lengths $L(\mathrm{~B})$ and unfolding forces $F(\mathrm{C})$.

Based on the previous research on disulfide bound proteins [20] and present MD simulations we propose 


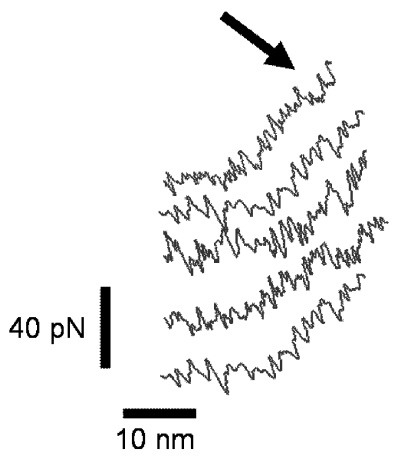

Fig. 3. Small force plateaus present in many human contactin 4 force-distance curves.

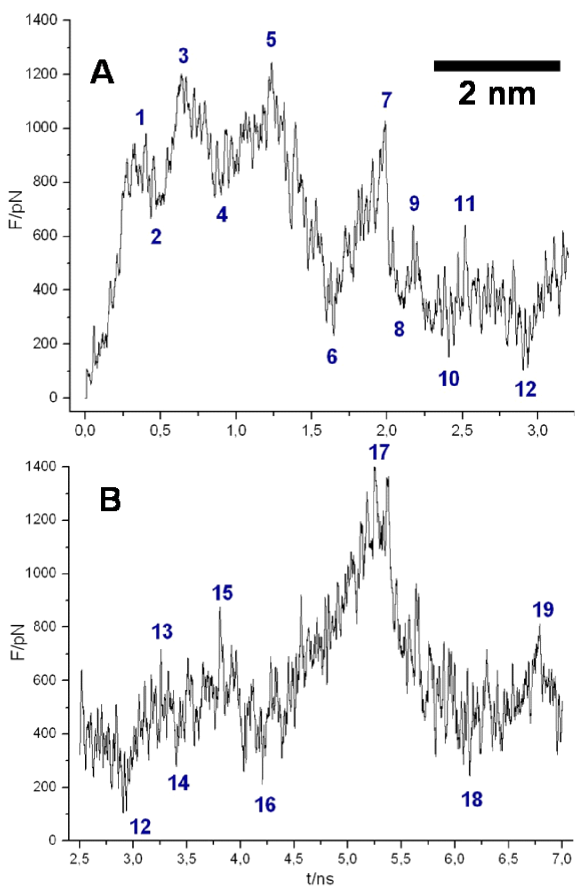

Fig. 4. The force-time (extension) curve for FNIII3 domain of CNTN4 model calculated by the SMD method. Numbered events are described in the text.

that the $19.41 \pm 1.63 \mathrm{~nm}$ centered peak results from the partial unfolding of $\mathrm{IgC} 2$ domains, while the remaining peaks are, respectively, intermediate and native unfolding events.

In some force curves a noticable drop of unfolding force was present, which has always appeared at the top in the first unfolding peak (Fig. 3). It bears some similarities to a hump observed during unfolding of titin molecules.[21]. A likely cause of this behavior is a rapid transition within the fold, resulting in an increase in length with a decreasing force.

An example of SMD unfolding process calculated for the domain FNIII3 (15 ns) is presented in Fig. 4A,B. All events 1-19 indicated in these spectra may be easily interpreted using computer graphics. Detailed analysis

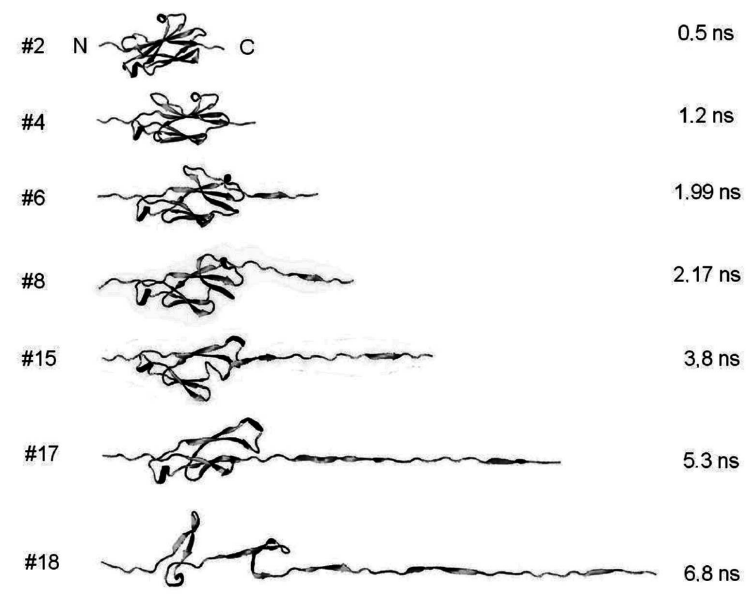

Fig. 5. Mechanical unfolding steps of FNIII3 domain predicted by SMD.

will be published elsewhere; here we only note that major drops in the exerted force are usually related with simultaneous breaking of a few hydrogen bonds between beta strands. A decrease of force in the 5, 6 and 7 region, resulting from breaking of hydrogen bonds between $G$ and $F$ strands provides a possible explanation for force drop from Fig. 3. Interestingly, during the mechanical unfolding new, "unnatural" hydrogen bonds are transiently formed. Time evolution (roughly proportional to the extension) is presented in Fig. 5 .

One should note that the direct comparison between the SMD and AFM results is difficult: the calculated force maxima (Fig. 4) are way too high due to the well known effect of the high pulling speed in SMD and the present data refer only to a one of the domains. SMD calculations for the full CNTN4 model are in progress.

\section{Conclusions}

Successful AFM stretching experiments were performed on single molecules of contactin 4. A higher resolution allowed us to use WLC analysis and observe small force plateaus during unfolding, previously not detected. A computer model of typical FNIII3 domain was constructed and $15 \mathrm{~ns}$ forced unfolding gave detailed atomistic picture of this process.

\section{Acknowledgments}

J. Strzelecki acknowledges grant "Krok w przyszłość" from Marszałek of Kujawsko-Pomorskie Voivodeship.

\section{References}

[1] T. Kaneko-Goto, S. Yoshihara, H. Miyazaki, Y. Yoshihara, Neuron 57, 834 (2008).

[2] T. Fernandez, T. Morgan, N. Davis, A. Klin, A. Morris, A. Farhi, R.P. Lifton, M.W. State, Am. J. Human Gen. 74, 1286 (2004). 
[3] J. Roohi, C. Montagna, D.H. Tegay, L.E. Palmer, C. DeVincent, J.C. Pomeroy, S.L. Christian, N. Nowak, E. Hatchwell, J. Med. Genet. 46, 176 (2009).

[4] G. Binnig, C.F. Quate, C. Gerber, Phys. Rev. Lett. 56, 930 (1986).

[5] E.L. Florin, V.T. Moy, H.E. Gaub, Science 264, 415 (1994).

[6] H. Clausen-Schaumann, M. Rief, C. Tolksdorf, H.E. Gaub, Biophys. J. 78, 1997 (2000).

[7] P.E. Marszalek, A.F. Oberhauser, Y.P. Pang, J.M. Fernandez, Nature 396, 661 (1998).

[8] G. Lee, W. Nowak, J. Jaroniec, Q. Zhang, P.E. Marszalek, Biophys. J. 87, 1456 (2004).

[9] M. Rief, M. Gautel, F. Oesterhelt, J.M. Fernandez, H.E. Gaub, Science 276, 1109 (1997).

[10] G. Lee, K. Abdi, Y. Jiang, P. Michaely, V. Bennett, P. Marszalek, Nature 440, 246 (2006).

[11] K. Lebed, M. Lekka, J. Lekki, W.M. Kwiatek, A. Dabrowska, J. Phys. Condens. Matter 18, 10157 (2006).

[12] J. Roohi, C. Montagna, D.H. Tegay, L.E. Palmer, C. DeVincent, J.C. Pomeroy, S.L. Christian, N. Nowak, E. Hatchwell, Brit. Med. J. 336, 414 (2008).

[13] M. Rabbi, P.E. Marszalek, Cold Spring Harbor Protocols 2007, pdb.prot4899, 2007.
[14] J.L. Hutter, J. Bechhoefer, Rev. Sci. Instrum. 64, 1868 (1993).

[15] C. Bustamante, J.F. Marko, E.D. Siggia, S.B. Smith, Science 265, 1599 (1994).

[16] M.T. Nelson, W. Humphrey, A. Gursoy, A. Dalke, L.V. Kale, R.D. Skeel, K. Schulten, Int. J. High Perform. Comput. Appl. 10, 251 (1996).

[17] T. Schwede, J. Kopp, N. Guex, M.C. Peitsch, Nucleic Acids Res. 31, 3381 (2003).

[18] W. Nowak, P. Marszalek, in: Current Trends in Computational Chemistry, Ed. J. Leszczyński, World Scientific, Singapore 2005.

[19] W. Humphrey, A. Dalke, K. Schulten, J. Mol. Graph. 14, 33 (1996).

[20] A.P. Wiita, S.R.K. Ainavarapu, H.H. Huang, J.M. Fernandez, Proc. Nat. Acad. Sci. 103, 7222 (2006).

[21] P. Marszalek, H. Lu, H. Li, M. Carrion-Vazquez, A. Oberhauser, K. Schulten, J. Fernandez, Nature 402, 100 (1999). 